\title{
External stenting: A reliable technique to relieve airway obstruction in small children
}

\author{
Makoto Ando, MD, ${ }^{\mathrm{a}}$ Yuzo Nagase, $\mathrm{MD},{ }^{\mathrm{b}}$ Hisaya Hasegawa, $\mathrm{MD},{ }^{\mathrm{c}}$ and Yukihiro Takahashi, $\mathrm{MD}^{\mathrm{a}}$
}

\section{ABSTRACT}

Objective: Airway obstruction in children may be caused by conditions such as vascular compression and congenital tracheobronchomalacia. Obstructive pulmonary vascular disease may be a detrimental sequel for patients with congenital heart disease. We evaluate our own original external stenting technique as a treatment option for these patients.

Methods: Ninety-eight patients underwent external stenting (1997-2015). Cardiovascular anomalies were noted in $82(83.7 \%)$. Nine patients had hypoplastic left heart syndrome and 6 had other types of single-ventricular hearts.

Results: The median age at the first operation was 7.2 months (range, 1.077.1 months). The mechanisms were tracheobronchomalacia with $(n=46)$ or without $(\mathrm{n}=52)$ vascular compression. Patients underwent 127 external stentings for 139 obstruction sites ( 62 trachea, 55 left bronchus, and 22 right bronchus). The stent sizes varied from 12 to $16 \mathrm{~mm}$. There were 14 ( 8 in the hospital and 6 after discharge) mortality cases. Nine required reoperation for restenosis and 3 required stent removal for infection. The actuarial freedom from mortality and any kind of reoperation was $74.7 \% \pm 4.6 \%$ after 2.8 years. The negative pressure threshold to induce airway collapse for congenital malacia $(\mathrm{n}=58)$ improved from -15.9 to $-116.0 \mathrm{cmH}_{2} \mathrm{O}$. A follow-up computed tomography scan ( $>2.0$ years interval from the operation; $\mathrm{n}=23$ ) showed the mean diameter of the stented segment at $88.5 \% \pm 13.7 \%$ (bronchus) and $94.5 \% \pm 8.2 \%$ (trachea) of the reference.

Conclusions: External stenting is a reliable method to relieve airway compression for small children, allowing an age-proportional growth of the airway. (J Thorac Cardiovasc Surg 2017;153:1167-77)

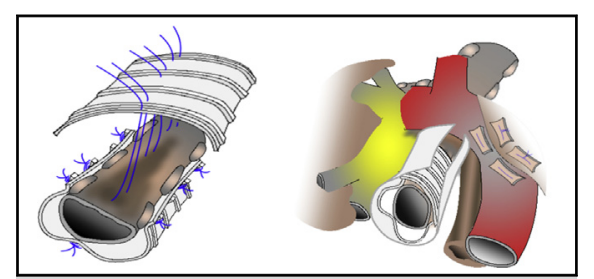

The scheme of the external stent technique using a ringed polytetrafluoroethylene graft.

\section{Central Message}

External stenting, suspending the airway to the surrounding rigid prosthesis, is a reliable method to relieve airway compression for small children.

\section{Perspective}

External stenting, suspending the airway to 2 separate pieces of rigid prosthesis, is a reliable method to relieve airway obstruction in small children. This is equally effective for tracheobronchomalacia with or without vascular compression, and may allow age-proportional growth of the airway. This technique may provide an alternative or adjunct to established practices such as aortopexy.

See Editorial Commentary page 1178
Severe airway obstruction in children may result from a congenital cartilage ring, intrinsic tracheobronchomalacia (TBM) or vascular compression syndrome. ${ }^{1-3}$ Many of these patients, especially with vascular compression, have coexisting congenital heart diseases. Even if the patient does not exhibit a life-threatening hypoxic spell, secondary pneumonia and atelectasis may lead to obstructive pulmonary vascular disease. ${ }^{4}$ Therefore, for the maintenance of

\footnotetext{
From the a Sakakibara Heart Institute; 'Ginza Heart Clinic; and ${ }^{\mathrm{c}}$ Tokyo Women's Medical University Medical Center East, Tokyo, Japan.

Read at the 96th Annual Meeting of The American Association for Thoracic Surgery, Baltimore, Maryland, May 14-18, 2016.

Received for publication May 5, 2016; revisions received Dec 5, 2016; accepted for publication Dec 15, 2016; available ahead of print Feb 24, 2017.

Address for reprints: Makoto Ando, MD, Department of Pediatric Cardiac Surgery, Sakakibara Heart Institute, 3-16-1 Asahi-cho, Fuchu-shi, Tokyo 183-0003, Japan (E-mail: maando@shi.heart.or.jp).

$0022-5223 / \$ 36.00$

Copyright (c) 2017 by The American Association for Thoracic Surgery http://dx.doi.org/10.1016/j.jtcvs.2016.12.041
}

cardiopulmonary function, the obstruction should be relieved at the earliest age possible.

An aortopexy offers a reasonable surgical option for TBM and vascular compression, but may not always assure airway expansion. An external stenting (ES)—-suspending the airway to the externally placed rigid prosthesis-may be an alternative option, ${ }^{6}$ but this prosthesis could interfere with the airway growth in small children. We herein evaluated the outcomes of our own original ES technique, using 2 separate pieces of ringed polytetrafluoroethylene (PTFE)

Scanning this QR code will take you to a supplemental video for the article. To view the AATS 2016 Webcast, see the URL next to the video thumbnail. 


$$
\begin{aligned}
& \text { Abbreviations and Acronyms } \\
& \text { CT = computed tomography } \\
& \text { ES = external stenting } \\
& \mathrm{LB}=\text { left bronchus } \\
& \text { PFTE }=\text { polytetrafluoroethylene } \\
& \mathrm{RB}=\text { right bronchus } \\
& \mathrm{T}=\text { trachea } \\
& \mathrm{TBM}=\text { tracheobronchomalacia }
\end{aligned}
$$

prosthesis with expectation of age-proportional growth of the airway.

\section{PATIENTS AND METHODS}

From April 1997 to July 2015, 98 patients underwent a total of 127 ES procedures. There were 43 female and 55 male patients. This was a 2-center study composed of 47 patients operated at Matsuda Municipal Hospital by the second author (Y.N.) and the remaining 51 patients at Sakakibara Heart Institute by the first author. The bronchoscopic evaluation was performed by the third author (H.H.) for all 98 patients. The mechanisms of airway obstruction were congenital TBM with $(n=46)$ or without vascular compression $(n=52)$. The operations were indicated due to severe hypoxic (dying) spells $(\mathrm{n}=36)$, failure to wean from the ventilator $(\mathrm{n}=34)$, respiratory distress requiring continuous or bilevel positive pressure breathing $(n=18)$ and recurrent respiratory infections $(n=10)$. The hypoxic spells were typically associated with tracheomalacia. Before undergoing operation, the details of the obstruction were assessed using a videoassisted flexible bronchoscopy and computed tomography (CT) scan. The median birth weight was $2.7 \mathrm{~kg}$ (range, 0.7-4.4 kg). Cardiovascular anomalies were noted in 82 patients $(83.7 \%$ ) (Table 1). Surgical corrections for these abnormalities were attempted for all patients. A total of 103 ES procedures were performed for these 82 patients. ES procedures were performed before $(n=39)$, simultaneously with $(n=25)$, or after $(n=39)$ the final repair. Patients with arch obstruction and 2 ventricles underwent direct anastomosis of the arch. Patients with hypoplastic left

\begin{tabular}{|c|c|}
\hline Operation & $\mathbf{n}$ \\
\hline $\begin{array}{l}\text { Pivotal operation performed prior to the first external } \\
\text { stenting }\end{array}$ & $\mathrm{n}=46$ \\
\hline Intracardiac biventricular repair & 15 \\
\hline Norwood operation & 8 \\
\hline $\begin{array}{l}\text { Aortic arch repair with intracardiac biventricular } \\
\text { repair }\end{array}$ & 8 \\
\hline Pulmonary arterial banding & 7 \\
\hline Systemic-to-pulmonary arterial shunt & 3 \\
\hline Glenn operation & 2 \\
\hline Bilateral pulmonary arterial banding & 1 \\
\hline Patent ductus arteriosus ligation & 1 \\
\hline Vascular ring repair & 1 \\
\hline $\begin{array}{l}\text { Operation performed simultaneously with the first } \\
\text { external stenting }\end{array}$ & $\mathrm{n}=24$ \\
\hline Intra-cardiac biventricular repair & 15 \\
\hline Vascular ring repair & 5 \\
\hline Aberrant innominate artery repair & 1 \\
\hline Patent ductus arteriosus ligation & 1 \\
\hline Systemic-to-pulmonary arterial shunt & 1 \\
\hline $\begin{array}{l}\text { Aortic arch repair with intracardiac biventricular } \\
\text { repair. }\end{array}$ & 1 \\
\hline Operation performed after the first external stenting & $\mathrm{n}=3$ \\
\hline Second-time external stenting (planned) & 18 \\
\hline Intracardiac biventricular repair & 9 \\
\hline Glenn operation & 1 \\
\hline Fontan operation & 1 \\
\hline Pulmonary arterial banding & 1 \\
\hline Second operation after the first stenting & $\mathrm{n}=6$ \\
\hline Intracardiac biventricular repair & 3 \\
\hline Third-time external stenting (planned) & 2 \\
\hline $\begin{array}{l}\text { Second-time external stenting (planned) with } \\
\text { intracardiac biventricular repair }\end{array}$ & 1 \\
\hline
\end{tabular}
heart syndrome underwent the Norwood operation except for 1 , who died after bilateral pulmonary arterial banding. Operations performed on these patients are listed in Table 2. There were 39 patients with chromosomal

TABLE 1. Cardiovascular anomalies

\begin{tabular}{lr}
\hline \multicolumn{1}{c}{ Cardiovascular anomaly } & $\mathbf{n}$ \\
\hline Ventricular septal defect & 22 \\
Hypoplastic left heart syndrome or variants & 9 \\
Arch obstruction with a heart with two ventricles & 9 \\
Atrial septal defect with or without anomalous pulmonary & 6 \\
$\quad$ venous connection & \\
Functionally single ventricle other than hypoplastic left heart & 6 \\
$\quad$ syndrome & 5 \\
Complete atrioventricular septal defect & 4 \\
Double aortic arch & 4 \\
Transposition of the great arteries & 4 \\
Absent pulmonary valve syndrome & 3 \\
Tetralogy of Fallot or double outlet right ventricle & 2 \\
Patent ductus arteriosus & 2 \\
Right aortic arch with left subclavian artery and ligamentum & 1 \\
Pulmonary artery sling & 5 \\
Others & 82 \\
Total &
\end{tabular}

TABLE 2. Operations performed for cardiovascular anomalies and airway obstructions

abnormality, including Down syndrome $(\mathrm{n}=17), 22 \mathrm{q} 11$ deletion $(\mathrm{n}=5)$, asplenia $(n=2)$, polysplenia $(n=2)$, Jacobsen syndrome $(n=2)$, SmithLemli-Opitz syndrome $(\mathrm{n}=1)$, Smith-Magenis syndrome $(\mathrm{n}=1)$, trisomy $8(\mathrm{n}=1)$, Turner syndrome $(\mathrm{n}=1)$, and Weaver syndrome $(\mathrm{n}=1)$ with 6 others having unspecified multiple organ malformations. Eight patients had undergone an unsuccessful aortopexy in the referring hospital. None of the remaining patients had undergone operations related to the airway.

Negative pressure testing - to measure the threshold at which airway collapse is induced - was performed before and after 58 ES procedures for TBM. This testing was performed under general anesthesia. The airway was observed using a bronchoscope, which was connected by a rubber tube to a vacuum system and a watertight $U$ tube. The water level of this tube was considered to reflect the amount of negative pressure imposed on the airway. A follow-up CT scan was performed in 23 patients (10 trachea [T] and 13 right bronchus [RB] or left bronchus $[\mathrm{LB}]$; maximum age $=8.7$ years) and the mean diameter of the stented area on the coronal and saggital planes were obtained. These were all patients who visited our outpatient clinic from March to July 2014 for follow-up. The inclusion criterion was a minimum of 2 years interval from the ES. Hence, there were no bias (ie, presence of a respiratory symptom) in selecting these patients. As a reference for $\mathrm{T}$ (mean age, $6.5 \pm 1.9$ years), a CT scan was performed for 11 age-matched volunteers $(6.2 \pm 0.2$ years; $P=.26)$. For the RB and LB, the maximal diameter of the main bronchus for the same patient was used. 
The end point of the study included mortality, reoperation, and removal of the mechanical ventilator or tracheostomy tube. The last date of data harvesting was July 2015 and the median follow-up period was 6.0 years (range, 0.1-18.2 years). The number of surviving patients ( 84 in total) being followed at the operating hospital (Matsuda Municipal Hospital or Sakakibara Heart Institute) was 57 with the remaining 27 followed at the referring hospital. The survival of these patients was confirmed by a questionnaire or direct contact with patients or referring hospitals. The institutional review board of Sakakibara Heart Institute approved the study and the procedures were in accordance with institutional guideline for protection of patient confidentiality. In the cases of Matsuda Municipal Hospital, a consent from was given to the volunteers of the CT study.

Details of the ES procedure are shown in Figure 1 as illustrations, in Video 1, and in Figure 2 as photographs. The basic surgical approach for a normal topography included left thoracotomy for the LB and a right thoracotomy for the $\mathrm{T}$ and the RB. Median sternotomy was chosen when a simultaneous cardiac procedure was required. Among 127 ES procedures performed on our patients, a left thoracotomy was for 52, right thoracotomy for 49 , and median sternotomy for 26 procedures. When the ascending or descending aorta was compressing the airway, the suspension of these vessels was performed simultaneously (60 procedures; $47.2 \%$ ). Usually, the ascending aorta was suspended to the sternum, and the descending aorta to the posterior part of the rib. Dissection of the airway is limited to the obstructed area, always attempting to minimize sacrifice of the feeding arteries. After the exposure of the obstructed airway, a bronchoscopy is performed to examine the site and extension of the obstruction. An endotracheal tube, if in place, is withdrawn to remove its splinting effect. A ringed PTFE prosthesis (Propaten; W.L. Gore \& Associates, Inc. Newark, Del) is then cut to a length corresponding to that of the obstructed airway segment. This usually involves 3 to 5 plastic rings. This is further cut longitudinally creating 2 semicircular pieces: a larger semicircle for the stabilization of the cartilage and a smaller semicircle for the membrane. The cartilage sutures are placed along 3 lines: midline and both lateral ends. To avoid air leak and infection, the bite of these stitches should be firm but not transmural. The midline stitches are firstly placed and passed through the graft. The graft is then placed around the airway and the sutures tied down. The redundant ends of the graft are trimmed off and lateral ends of the cartilage sutured to the graft. It is important to ensure that the graft forms a smooth arch leaving a reasonable space around, but not to an extent that distorts the airway. The other graft piece is fixed to the membrane by stitches placed along the midline. Both ends of the membrane stent are then trimmed off so that it overlaps the cartilage stent by a few millimeters. It is imperative that these 2 prosthetic segments are not sutured together. The final bronchoscopy was performed after the procedure with the patient in a supine position. At this time, the end-expiratory pressure was kept at zero on the ventilator setting.
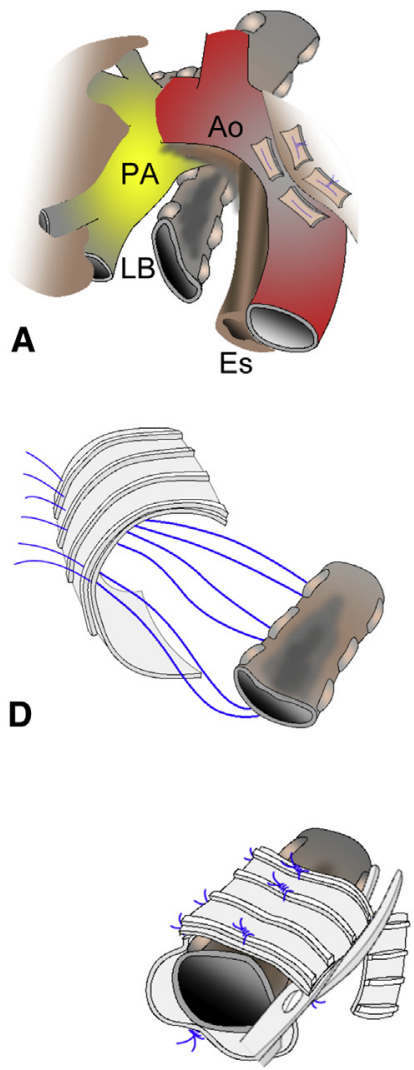

G

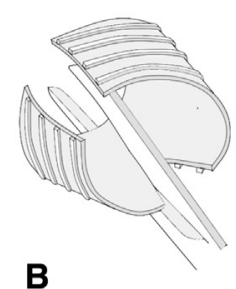

B

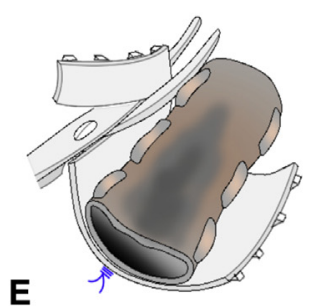

$\mathbf{E}$

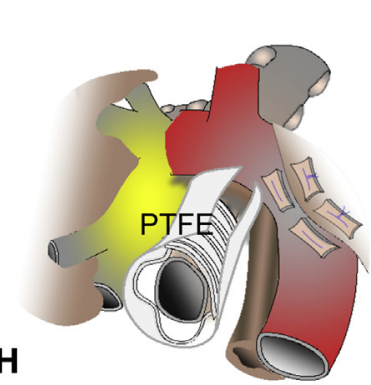

C

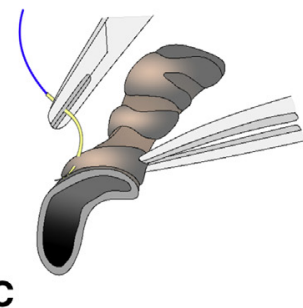

(

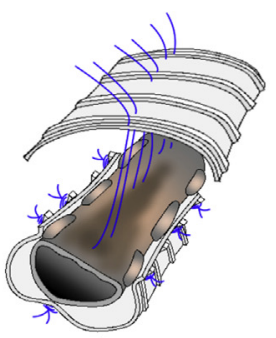

FIGURE 1. The scheme of the external stent technique for the left bronchial compression by the descending aorta. A, The aorta is first retracted away from the airway towards the chest wall using pledgeted sutures. B, A ringed polytetrafluoroethylene prosthesis is cut into 2 semicircular pieces. C, The midline stitches are placed. D, The midline stitches are passed through the graft. E, The redundant ends of the graft are trimmed off. F, The lateral ends of the cartilage sutured to the graft. The other graft piece is fixed to the membrane by stitches placed along the midline. G, Both ends of the membrane stent are then trimmed off. $\mathrm{H}$, The grafts are finally covered by a $0.1-\mathrm{mm}$ polytetrafluoroethylene sheet to prevent esophageal erosion. Ao, Aorta; $P A$, pulmonary artery; $L B$, left bronchus; Es, esophagus. 

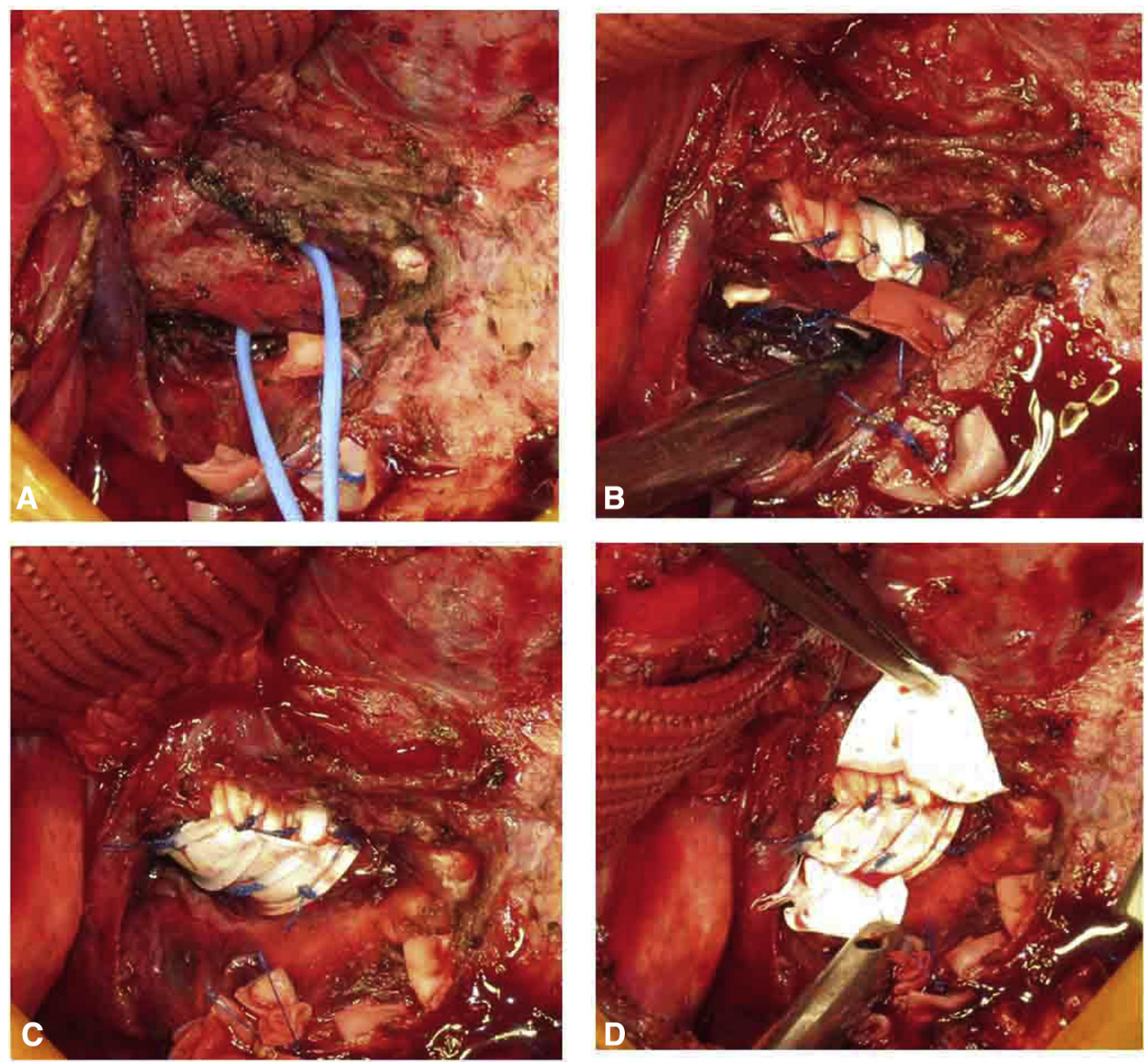

FIGURE 2. The operative photos of the external stenting for the left bronchus through a left thoracotomy. A, The cartilage portion is stabilized. B, The graft for membrane portion is passed around the airway. C, The fixation of the membrane portion further expands the airway. D, The stent is finally covered by a 0.1-mm polytetrafluoroethylene sheet.

Our ES technique might be modified to solve some specific situations. One, for example, is to use a single piece of graft-instead of 2-placed around the airway opposite the compressing vessel, leaving scar tissue in between. The retraction of the airway opposite the vessel would expand the airway because adhesion to the vessel exists (Figure 3).

\section{Data Analysis}

Data are presented as median or mean \pm standard deviation. Student $t$ test was used to analyze intergroup differences of continuous variables. Time-dependent outcomes were assessed with Kaplan-Meir analysis, with a long-rank test to evaluate group differences. Statistical analysis and graphic drawing was performed using JMP software for Macintosh, version 10.0.2 (SAS Institute Inc, Cary, NC).

\section{RESULTS}

The median age at the first ES was 7.2 months (range, 1.0-77.1 months), with 71 patients $(72.4 \%)$ younger than age 1 year. The obstruction site was $\mathrm{T}(\mathrm{n}=27), \mathrm{RB}$ $(\mathrm{n}=4), \mathrm{LB}(\mathrm{n}=32), \mathrm{T}+\mathrm{RB}(\mathrm{n}=12), \mathrm{T}+\mathrm{LB}$ $(n=17)$, and $T+R B+L B(n=6)$. For the lesion $\mathrm{T}+\mathrm{RB}$, the ES was either single- $(\mathrm{n}=5)$ or 2-staged $(\mathrm{n}=7)$. It was single- $(\mathrm{n}=2)$ or 2 -staged $(\mathrm{n}=15)$ for $\mathrm{T}+\mathrm{LB}$, and single- $(\mathrm{n}=1), 2-(\mathrm{n}=3)$ or 3-staged $(\mathrm{n}=2)$ for $\mathrm{T}+\mathrm{RB}+\mathrm{LB}$. Hence, patients underwent a total of 127 ES procedures for these 139 obstruction sites. The mode of vascular compression is summarized in Figure 4. The graft sizes were $16 \mathrm{~mm}(\mathrm{n}=51)$ and $14 \mathrm{~mm}$ $(\mathrm{n}=11)$ for $\mathrm{T}$; $16 \mathrm{~mm}(\mathrm{n}=14), 14 \mathrm{~mm}(\mathrm{n}=5)$, and $12 \mathrm{~mm}(\mathrm{n}=3)$ for $\mathrm{RB}$; and $16 \mathrm{~mm}(\mathrm{n}=33), 14 \mathrm{~mm}$ $(\mathrm{n}=9)$, and $12 \mathrm{~mm}(\mathrm{n}=13)$ for LB.

There were 8 in-hospital and 6 postdischarge mortalities. The leading cause was congestive heart failure in 6 patients: 2 died immediately after operation due to severe ventricular dysfunction, the other 4 died from an ensuing cardiac dysfunction with immune dysfunction predisposing to infectious complication. The other causes of mortality included gastrointestinal bleeding $(\mathrm{n}=3)$, respiratory distress $(\mathrm{n}=3)$, hepatoblastoma $(\mathrm{n}=1)$, and a traffic accident $(\mathrm{n}=1)$. The actuarial survival was $84.2 \% \pm 4.0 \%$ after 1.8 years. In particular, 12 out of 14 mortalities had an associated heart disease. Also noteworthy, the mortality rate reached $30.4 \%$ (7 out of 23 patients) when both tracheal and left bronchial obstructions were seen simultaneously. On the other hand, the mortality rate was $9.3 \%$ 


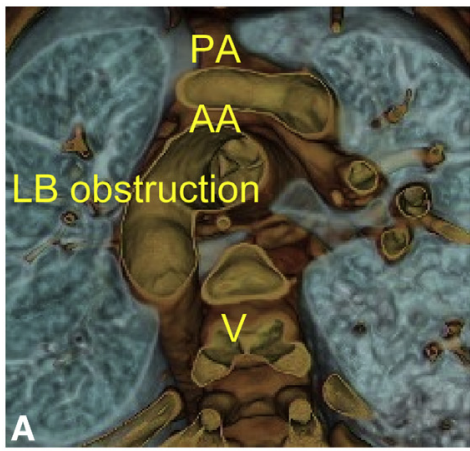

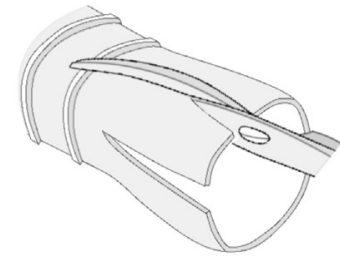

B

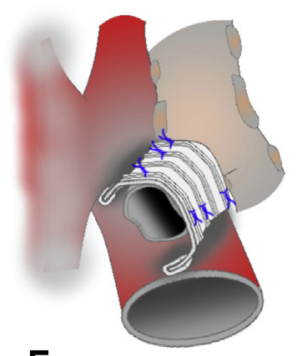

E

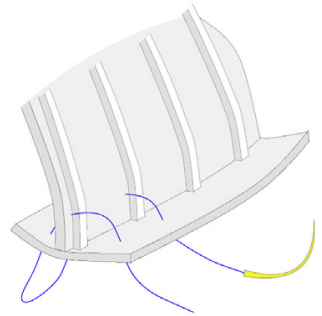

C
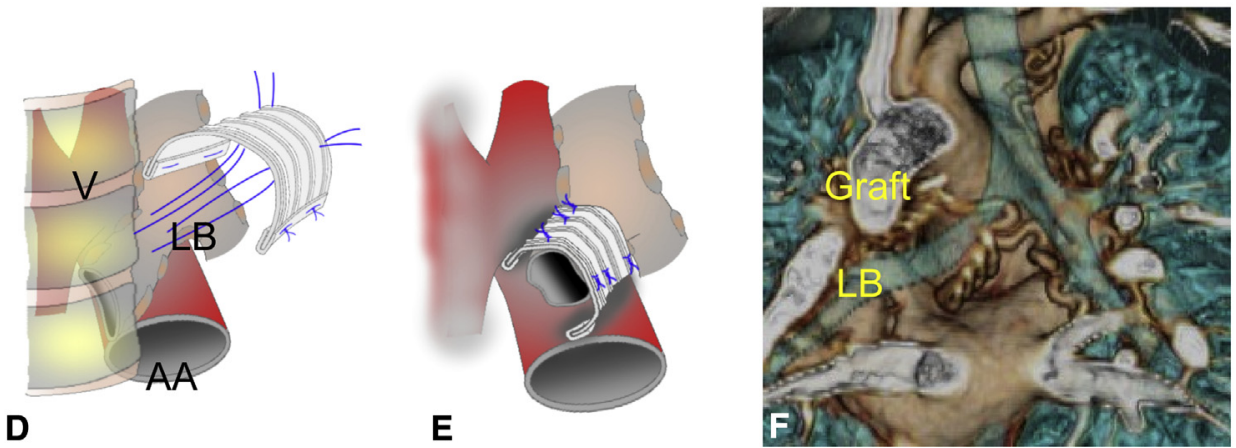

FIGURE 3. The scheme of a modified external stent technique used in a patient who had undergone an arterial switch operation. A, On the preoperative computed tomography scan, the aorta was displaced dorsally, compressing the left bronchus $(L B)$ flatly against the chest wall and left no space to allow placement of 2 graft pieces. B, In this case, a single piece of graft—instead of 2-was placed around the airway opposite to the ascending aorta, leaving a scar tissue in between. B and C, The graft bore protecting cuffs, made of the same graft, to prevent vascular erosion. D, Sutures were placed on the membrane portion. E, The retraction of the membrane portion opposite the aorta expanded the airway as adhesion to the vessel existed. F, The postoperative computed tomography scan showed an expanded LB. PA, Pulmonary artery; $A A$, ascending aorta; $V$, vertebral column.

(7 out of 75) when the obstruction was confined to the single site on either $\mathrm{T}$ or $\mathrm{RB}$ or $\mathrm{LB}$, or when the $\mathrm{T}$ and the $\mathrm{RB}$ were simultaneously involved.

Nine patients required the ES to be redone at the median of 1.0 month (range, 0.1-14.4 months) after the initial operation for persistent or recurrent obstruction. At the time of reoperation, the former stent was removed and replaced by a new stent under bronchoscope guidance. The actuarial freedom from reoperation for restenosis among the surviving patients was $90.1 \% \pm 3.2 \%$ after 1.2 years. This number did not differ between those with $(90.1 \% \pm 4.7 \%)$ and without vascular compression $(90.1 \% \pm 4.2 \% ; P=.995)$. In 5 patients, full expansion of the airway was not obtained after the first operation and respiratory symptoms persisted. In the other 4 patients, vascular compression recurred despite being effectively retracted in the initial operation. Even after the repeat ES, 2 patients with a bronchial obstruction required a third ES, with 1 of those even requiring a fourth. In the case of this latter patient, the compression from the aorta persisted and we could not achieve complete expansion of the airway. Three other patients required stent removal due to perforation into the airway followed by infection occurring at the carina $(\mathrm{n}=2)$ and at the origin of the upper lobe bronchus. For these patients, the graft was removed and the hole was repaired by a glutaraldehyde-fixed pericardium at 1.2, 1.8, and 2.7 years after the ES. The infections resolved thereafter and these airways have stayed patent without further interventions. The actuarial freedom from mortality and any kind of reoperation was $74.7 \% \pm 4.6 \%$ after 2.8 years (Figure 5 ).

The other major postoperative complications included phrenic nerve paralysis requiring diaphragmatic plication $(\mathrm{n}=2)$, bilateral recurrent laryngeal nerve injury requiring tracheostomy $(\mathrm{n}=1)$, esophageal perforation $(\mathrm{n}=1)$, mediastinitis $(\mathrm{n}=1)$, pulmonary venous compression $(\mathrm{n}=1)$, and lung bleeding resulting from acute respiratory syndrome. Esophageal perforation developed in a patient with hypoplastic left heart syndrome. After the ES of the LB the stent presumably eroded into both the descending aorta and esophagus. This resulted in aortoesophageal fistula and death due to massive bleeding. The pulmonary venous 

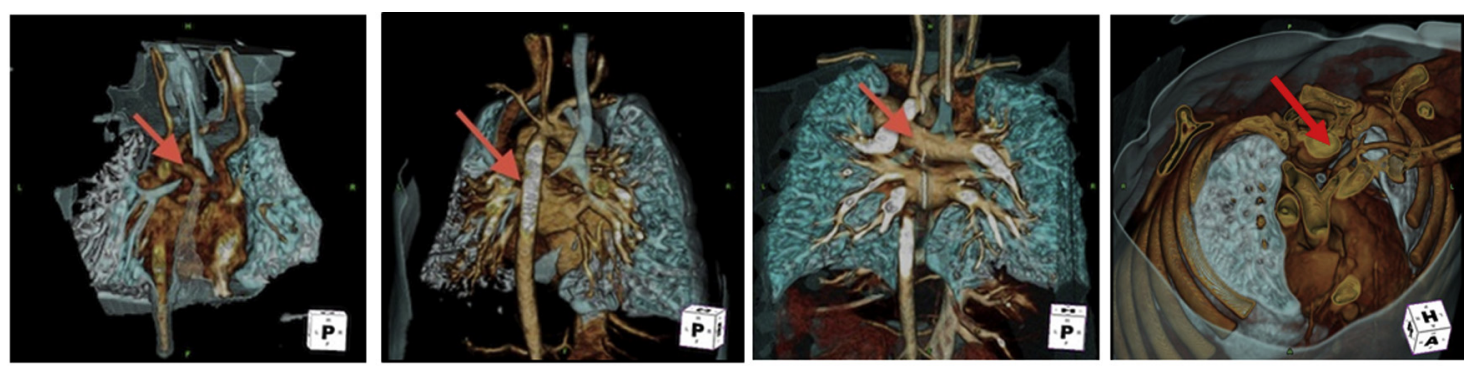

\begin{tabular}{|l|c|c|c|c|}
\hline & $\begin{array}{c}\text { Ascendingaorta or aortic } \\
\text { arch }\end{array}$ & $\begin{array}{c}\text { Descending aorta } \\
\text { Pulmonary artery }\end{array}$ & $\begin{array}{c}\text { Innominate or subclavian } \\
\text { artery }\end{array}$ \\
\hline Trachea & 11 & 0 & 2 & 3 \\
\hline Right bronchus & 4 & 0 & 3 & 0 \\
\hline Left bronchus & 2 & 24 & 2 & 0 \\
\hline & 17 & & & 3 \\
\hline Total & 24 & 7 & 3 \\
\hline
\end{tabular}

FIGURE 4. Modes of vascular compression. The responsive vessel can be the descending aorta, pulmonary artery, ascending aorta/aortic arch or innominate/subclavian artery. The number of each vessel compressing the trachea, right bronchus, and left bronchus are listed.

compression resolved spontaneously with observation. In the patient who developed bilateral phrenic nerve injuries, the phrenic nerves were coursing between the $T$ and the compressing vessels with a dense adhesion caused by the prior aortopexy.

Information regarding the present respiratory status of the patients was not obtainable in 7 out of 84 survivors. In the remaining 77 patients, $69(89.6 \%)$ were weaned from the ventilator at a median of 5 days (range, 0-330 days) after the first ES. Presently, 11 out of these 77 patients (14.3\%) have a tracheostomy tube in place.

The negative pressure testing revealed the airway in patients with TBM remained patent until the imposed pressure reached $-116.0 \mathrm{cmH}_{2} \mathrm{O}$ after operation compared with $-15.9 \mathrm{cmH}_{2} \mathrm{O}$ before operation (Figure 6). The mean percent diameter was $88.5 \% \pm 13.7 \%$ for the bronchus and $94.5 \% \pm 8.2 \%$ for the $\mathrm{T}$ (Figure 7 ).

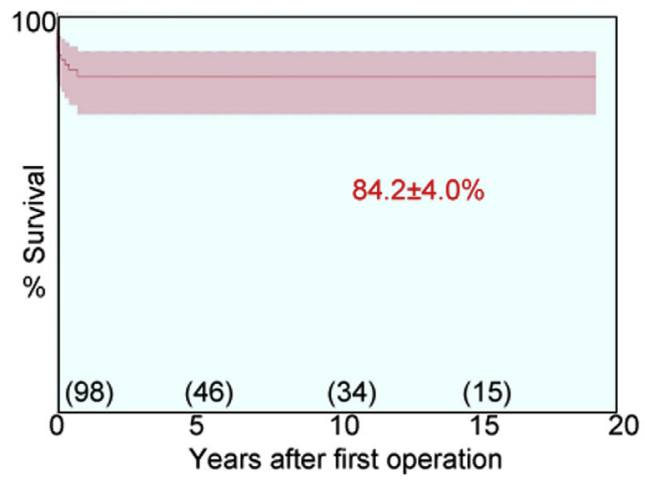

\section{DISCUSSION}

An airway obstruction in children may result from a cartilage ring, ${ }^{1}$ congenital $\mathrm{TBM},{ }^{2}$ vascular compression, ${ }^{3}$ and other reasons. Presently, sliding plasty is the gold standard for a tracheal ring. ${ }^{7,8}$ TBM may be an intrinsic problem but is also associated with vascular compression. ${ }^{9-13}$ These patients, especially with vascular compression, often have coexisting congenital cardiovascular anomalies. ${ }^{14}$ Isolated TBM may be observed without invasive treatment because spontaneous resolution can be expected. ${ }^{15}$ For patients with congenital heart disease, early intervention may be beneficial because obstructive pulmonary vascular disease may develop following recurrent pneumonia. $^{4}$

Aortopexy is considered a main stem of the surgical repair $^{16}$ for TBM with or without vascular compression. However, some report a high failure rate of aortopexy for

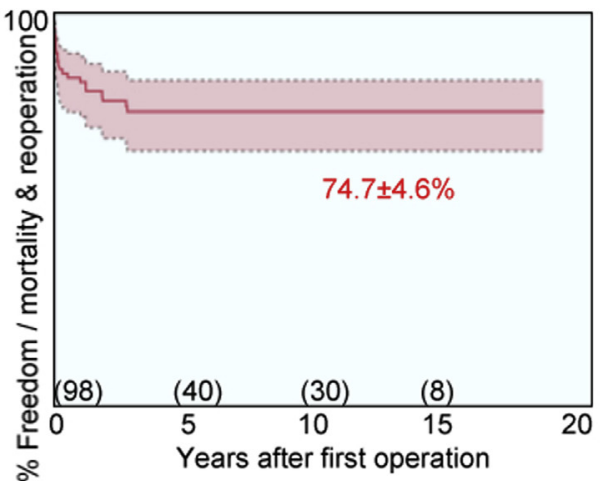

FIGURE 5. Actuarial survival and freedom from mortality and any kind of reoperation with a $95 \%$ confidence interval. Numbers in parentheses show patients at risk immediately, 5 years, 10 years, and 15 years after the first external stenting procedure. 


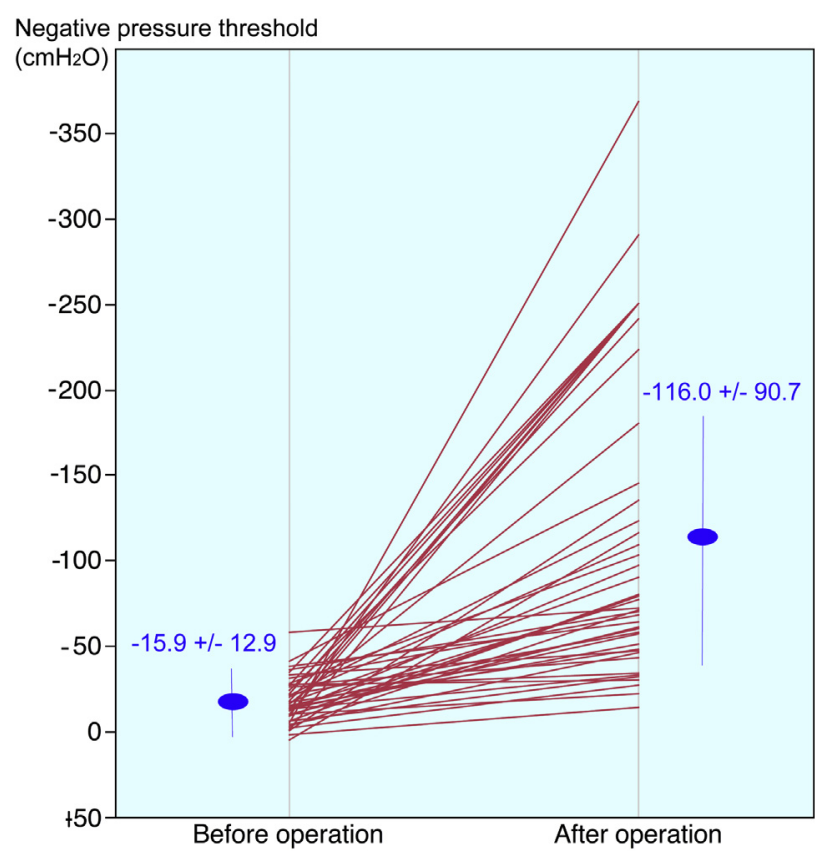

FIGURE 6. The parallel plot showing the results of negative pressure testing. Red bars indicate perioperative transition of the pressure threshold to induce airway collapse. The blue dot indicates the mean value with the standard deviation.

vascular compression due to an irreversible structural deformity of the cartilage. ${ }^{17}$ Also, retraction of the responsible artery may be hazardous: An example is an aortic arch

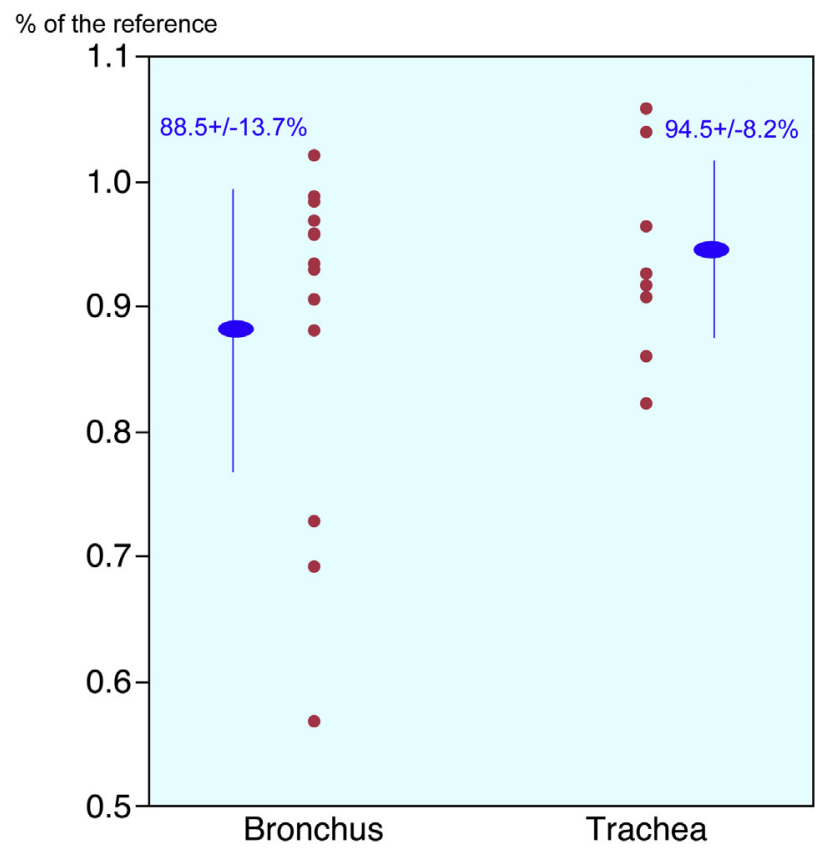

FIGURE 7. The scattergram of the airway diameter of the stented segment (the mean of those on the coronal and saggital views of the computed tomography scan) expressed as the percentage of the reference value. The blue dot indicates the mean value with the standard deviation. with its unique hairpin configuration and branching neck vessels. Another example is the retraction of the aortic arch after the arterial switch operation for transposition of the great arteries, which is difficult due to the anterior pulmonary artery. Other options include reconstruction surgeries; however, these techniques may be associated with recurrent obstruction due to granulation formation and anastomotic tension. ${ }^{18,19}$ Moreover, reports of these techniques performed for bronchi of small children are limited. $^{20,21}$ As a nonsurgical intervention, the internal splinting with an endotracheal tube may be an option, ${ }^{22}$ but the prolonged intubation may cause chronic inflammation of the airway. Endoscopic stent placement is also an option; however, the reported rate of complications and death ranges from $23.8 \%$ to $38.1 \%,{ }^{23,24}$ or perhaps higher with a vascular compression. ${ }^{25}$

ES is a unique surgical option to expand and stabilize the airway by suspending its wall to the surrounding rigid prosthesis. The mucous membrane is not disrupted, and therefore, granulation tissue formation is unlikely. To our knowledge, the first report of ES on a series of patients was published in $1997 .{ }^{6}$ According to that work, the ringed PTFE graft was longitudinally cut and resutured together after being passed around the airway. Considering the future airway growth, a large prosthesis $(20 \mathrm{~mm}$ for $\mathrm{T}$ and $16 \mathrm{~mm}$ for RB or LB) was used.

The second author (Y.N.) started using his own original technique of ES in 1997, unaware of the publication of this similar technique in the same year. ${ }^{26}$ In our technique, the graft segment is cut longitudinally into 2 pieces: the larger for stabilization of the cartilage portion, and the smaller for the membrane portion. Importantly, these 2 pieces are not sutured together, to allow the airway growth. This also enables us to use a smaller prosthesis $(12-16 \mathrm{~mm})$, which is especially important in young patients, thus avoiding compression of the important adjacent structures such as the pulmonary vein or esophagus. The choice of graft size follows measuring of the external airway diameter during the operation. In general, the use of a 16-mm graft may be considered if the airway diameter is $>7$ to $8 \mathrm{~mm}$. The 12 $\mathrm{mm}$ graft is usually chosen if the diameter is $<5 \mathrm{~mm}$. It is safer to use a smaller graft if the esophagus or pulmonary vein is in the vicinity. The graft usually involves 3 to 5 plastic rings and ideally should cover the entire area of the obstruction, although it could be somewhat shorter. In most of our patients, an age-proportional growth of the stented segment has been observed, despite a concern of fibrous tissue ingrowth. ${ }^{27}$

In normal topography, we consider the right thoracotomy to be the best approach for the T or RB and the left thoracotomy for the LB. A midline approach does not allow precise stitching on the membrane portion of the airways, which is on the dorsal aspect. Therefore, this approach is chosen only when a simultaneous cardiac procedure is required, or the 


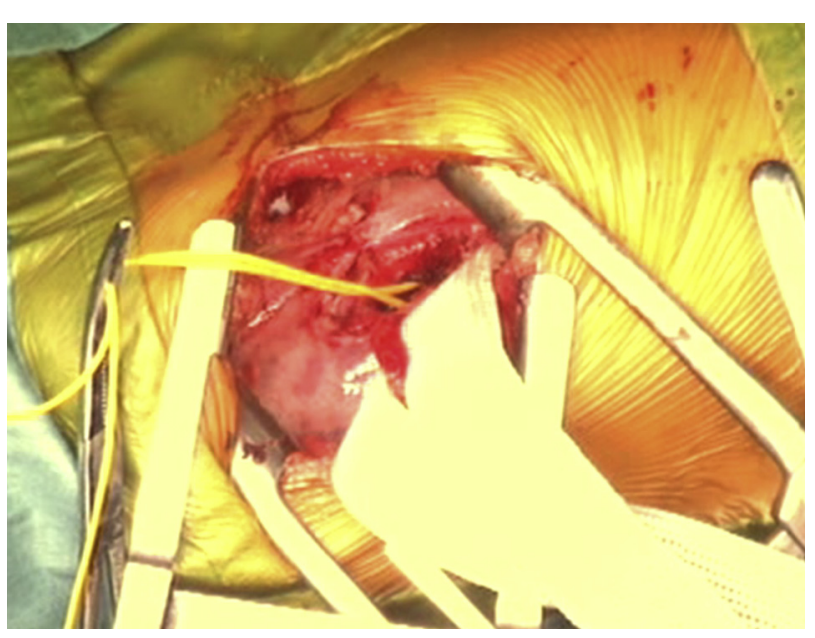

VIDEO 1. The surgical video of the external stenting for the left bronchus through a left thoracotomy. Video available at: http://www.jtcvsonline.org/ article/S0022-5223(17)30150-2/addons.

right side of the chest has previously been entered. Occasionally, multiple airway sites are involved, requiring multistaged operations. Usually the tracheal stent precedes bronchial stents. Occasionally, a bronchial stent was performed first if the obstruction was very severe.

Graft perforation into the airway is a serious complication of ES. Our results suggested 2 potential situations for this: a tracheal stent perforating into the main bronchus and the right main bronchial stent into the upper bronchus. TBM occasionally develops around the carina and the tracheal stent can be placed close to the carina. Also, the $\mathrm{RB}$ is often short and the stent may be placed close to the origin of the upper bronchus. Hence, any downstream migration of these grafts could cause perforation. Our current practice includes the use of a shorter graft pieces (mostly involving 3 rings) for these sites, leaving a distance between the distal end of the graft and the nearby bronchial origin.

Our experience also showed that compressive injury on the adjacent structure, such as esophagus or pulmonary vein, is possible and may become fatal. ${ }^{28}$ To avoid these complications, it is especially important to secure a generous space for the insertion of the graft pieces and maximal effort is required to remove the compressive effect of the responsible vessel. Also, ringed PTFE graft is a stiff material with sharp edges. We have, therefore, been placing a 0.1-mm PTFE sheet covering the entire area of these grafts as a precaution against graft erosion into neighboring important structures such as the esophagus.

Survival of small patients with airway obstruction requires comprehensive medical management not only for the airway, but also for other coexisting problems. Our series of operations, in fact, showed that more than $80 \%$ of patients had a congenital cardiovascular disorder and about
$40 \%$ had a chromosomal abnormality. Another difficult situation is when multiple-rather than single-airway segments are obstructed simultaneously. This, in itself, can suggest the complexity of the overall anatomy. Our study especially showed that the coexistence of tracheal and LB obstructions, often associated with an extensive and complex mode of compression by the aorta, might be prognostic of mortality.

Compressive effect of the vessel may remain despite maximal effort. This is especially a concern when the patient has a short and low aortic arch along with a small aortopulmonary window. In this case, placement of a rigid graft may further obliterate the window and an aortopexy might offer better results. In whichever case, it is possible that these vessels can revert to their former position because loosening of the retraction suture or chest deformity may occur after some time.

The main limitations of this study include its retrospective design, limited sample size, and short follow-up. Also, a significant percentage of patients are now being followed at outside hospitals because we commonly accept nationwide referrals. Therefore, obtained data included their survival and surgical history but no other details. It is imperative that the airway of these patients be examined when they reach adulthood-using CT scan or bronchoscopy - to ascertain the need for stent removal. And finally, this study does not provide any data for meaningful comparison of the ES with the other procedures such as aortpexy because we performed ES in the majority of our patients.

\section{CONCLUSIONS}

External stenting is a reliable method to relieve airway compression in small children. It is effective equally for TBM with or without vascular compression. It may allow age-proportional growth of the airway due to the noncircumferential and oversized design of the prosthesis. Our technique of ES may provide an alternative or adjunct to established practices such as aortopexy.

\section{Webcast}

You can watch a Webcast of this AATS meeting presentation by going to: http://webcast.aats.org/2016/Video/ Tuesday/05-17-16_Hall_E_0845_Ando-800.mp4.

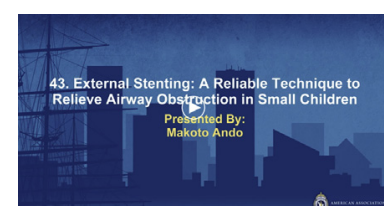

\section{Conflict of Interest Statement}

Authors have nothing to disclose with regard to commercial support. 


\section{References}

1. Berdon WE, Baker DH, Wung JT, Chrispin A, Kozlowski K, de Silva M, et al. Complete cartilage-ring tracheal stenosis associated with anomalous left pulmonary artery: the ring-sling complex. Radiology. 1984;152:57-64.

2. Cogbill TH, Moore FA, Accurso FJ, Lilly JR. Primary tracheomalacia. Ann Thorac Surg. 1983;35:538-41.

3. Koivikko A, Puhakka HJ, Vikki P. Innominate artery compression syndrome. Presentation of 16 cases. ORL J Otorhinolaryngol Relat Spec. 1976;38:187-92.

4. Kugler C, Stanzel F. Tracheomalacia. Thorac Surg Clin. 2014;24:51-8.

5. Emery JL, Nanayakkara CF, Wailoo MP. Tracheomalacia-lethal factor in a 17month-old child. Pediatr Pathol. 1984;2:259-65.

6. Hagl S, Jakob H, Sebening C, van Bodegom P, Schmidt K, Zilow E, et al. External stabilization of long-segment tracheobronchomalacia guided by intraoperative bronchoscopy. Ann Thorac Surg. 1997;64:1412-20.

7. Butler CR, Speggiorin S, Rijnberg FM, Roebuck DJ, Muthialu N, Hewitt RJ, et al. Outcomes of slide tracheoplasty in 101 children: a 17-year single-center experience. J Thorac Cardiovasc Surg. 2014;147:1783-9.

8. Grillo HC, Wright C, Vlahakes G, MacGillivray T. Management of congenital tracheal stenosis by means of slide tracheoplasty or resection and reconstruction, with long term follow-up of growth after slide tracheoplasty. J Thorac Cardiovasc Surg. 2002;123:145-52.

9. Gross RE, Neuhause EBD. Compression of the trachea by an anomalous innominate artery: an operation for its relief. Am Dis Child. 1948;75:570-4.

10. Lincoln JC, Deverall PB, Stark J, Aberdeen E, Waterston DJ. Vascular anomalies compressing the oesophagus and trachea. Thorax. 1969;24:295-306.

11. Baxter JD, Dunbar JS. Tracheomalacia. Ann Otol Rhinol Laryngol. 1963;72: 1013-23.

12. Blair GK, Cohen R, Filler R. Treatment of tracheomalacia: eight years' experience. J Pediatr Surg. 1986;21:781-5.

13. Sebening C, Jakob H, Tochtermann U, Lange R, Vahl CF, Bodegom P, et al. Vascular tracheobronchial compression syndromes-experience in surgical treatment and literature review. Thorac Cardiovasc Surg. 2000; 48:164-74.

14. Schreiber C, Mazzitelli D, Haehnel JC, Lorenz HP, Meisner H. The interrupted aortic arch: an overview after 20 years of surgical treatment. Eur J Cardiothorac Surg. 1997;12:466-9.

15. Antón-Pacheco JL, Cano I, García A, López M, Cabezalí D, Martínez A. Management of congenital tracheomalacia: a single institution experience. $\mathrm{Cir} \mathrm{Pe}$ diatr. 2006;19:55-60.

16. Abdel-Rahman U, Ahrens P, Fieguth HG, Kitz R, Heller K, Moritz A. Surgical treatment of tracheomalacia in bronchoscopic monitors aortopexy in infant and children. Ann Thorac Surg. 2002;74:315-9.

17. Filler RM, Rossello PJ, Lebowitz RL. Life threatening anoxic spells caused by tracheal compression after repair of esophageal atresia: correction by surgery. J Pediatr Surg. 1976;11:739-48.

18. Nakayama DK, Harrison MR, de Lorimier AA, Brasch RC, Fishman NH. Reconstructive surgery for obstructing lesions of the intrathoracic trachea in infants and small children. J Pediatr Surg. 1982;17:854-68.

19. Vinograd I, Filler RM, Bahoric A. Long-term functional results of prosthetic airway splinting in tracheomalacia and bronchomalacia. J Pediatr Surg. 1987; 22:38-41.

20. Waldhausen JH, Richardson M, Hall DG. A new technique for surgical correction of bronchial stenosis. J Pediatr Surg. 1994;29:199-200.

21. Filler RM, Messineo A, Vinograd I. Severe tracheomalacia associated with esophageal atresia: results of surgical treatment. J Peditar Surg. 1992;27: 1136-40.

22. Greenholz SK, Karrer FM, Lilly JR. Contemporary surgery of tracheomalacia. J Pediatr Surg. 1986;21:511-4.

23. Antón-Pacheco JL, Cabezalí D, Tejedor R, López M, Luna C, Comas JV, et al. The role of airway stenting in pediatric tracheobronchial obstruction. Eur $J$ Cardiothoracic Surg. 2008;33:1069-75.

24. Corno A, Giamberti A, Giannico S, Marino B, Rossi E, Marcelletti C, et al. Airway obstructions associated with congenital heart disease in infancy. J Thorac Cardiovasc Surg. 1990;99:1091-8.

25. Valerie EP, Durrant AC, Forte V, Wales P, Chait P, Kim PC. A decade of using intraluminal tracheal/bronchial stents in the management of tracheomalacia and/or bronchomalacia: is it better than aortopexy? J Pediatr Surg. 2005;40: 904-7.

26. Takazawa S, Uchida H, Kawashima H, Tanaka Y, Masuko T, Deie K, et al. External stabilization for severe tracheobronchomalacia using separated ring- reinforced ePTFE grafts is effective and safe on a long-term basis. Pediatr Surg Int. 2013;29:1165-9.

27. Ley S, Loukanov T, Ley-Zaporozhan J, Springer W, Sebening C, Sommerburg O, et al. Long-term outcome after external tracheal stabilization due to congenital tracheal instability. Ann Thorac Surg. 2010;89:918-25.

28. Schweigert M, Santos Sousa H, Solymosi N, Yankulov A, Fernández MJ, Beattie R, et al. Spotlight on esophageal perforation: a multinational study using the Pittsburgh esophageal perforation severity scoring system. J Thorac Cardiovasc Surg. 2016;151:1002-11.

Key Words: vascular compression syndrome, congenital heart disease, tracheomalacia, surgery

\section{Discussion}

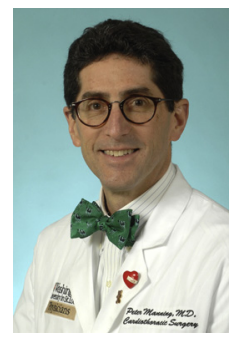

Dr Peter Manning (St Louis, Mo). Tracheobronchomalacia is an oftenfrustrating problem that we see commonly in children, often those with congenital complex heart defects.

Although the majority of cases will improve without any surgical intervention due to the growth of the airway both in size and sturdiness, there are certainly cases where a more aggressive approach is warranted due to the severity of associated symptoms.

The diversity of surgical approaches is testament to the fact that no single approach predictably yields good results. They can generally be grouped into internal support (tracheostomy and positive pressure ventilation, for example), or external support measures.

The external support strategies to move adjacent vascular structures have long been the mainstay of surgical management with external stents being reported previously in only a handful of case reports and small series.

The experience presented today by Dr Ando is valuable due to the number of cases in which they have performed external stenting and their acquisition of a reasonable amount of late follow-up in these cases.

The authors conclude that this is a less-invasive and reliable method to relieve airway compression. I have to disagree that it is less invasive than other strategies, and whereas the authors do show that the procedure works for many patients, it certainly is not a panacea, with more than $10 \%$ of patients still requiring mechanical ventilation nearly a year later. In addition, $10 \%$ of patients required early reoperation for what is described as restenosis.

Dr Ando, how did restenosis occur early following the original procedure, and what was done at reoperation in these cases?

Dr Ando. Thank you very much for your question. Indeed, any kind of procedure needs a learning curve, and at the beginning of the series, I failed in some cases.

And another thing is even if you can relieve the airway efficiently, there are some cases such as a very short aortic . 
arch or low aortic arch where you have a very small aortopulmonary window in which you can hardly place 2 pieces of graft. In that case, I would just pull the arch out of the airway as much as possible. However, when the patient starts moving around, maybe the stitch can loosen or break apart, or maybe there can be some kind of changes in topography between the vessels and the airway so that the patients comes back to the restenosis.

So I think the essence is how effectively you can remove the compression of the vessel as much as possible. In some cases, like what I said, it is very difficult. These are the very cases where we sometimes see the reobstruction.

Dr Manning. At the reoperation, were you able to redo the original operation, or did you have to go to a different strategy?

Dr Ando. Yes, I could. Very surprisingly, it is not tough to remove this stent. It just comes out without difficulty, even if it is right after the operation or several hours out of the operation.

Although it certainly has a denser adhesion than before, it makes the operation tougher. But as for the removal of the stent, we can repeat it as many as needed.

Dr Manning. Thank you. There were 3 cases of infection, which were all erosions into the airway that occurred between 1 and 3 years after the original procedure. With this approach, will we need to be concerned about the risk of this complication indefinitely? After all, this procedure is typically done in small children.

Do you suggest any form of ongoing evaluation of the patients to detect when this erosion may be developing before it perforates?

Dr Ando. About the infection you mean? Sorry, what is your question?

Dr Manning. Are you always going to be concerned about late erosion?

Dr Ando. All right. As for the infection, I think it can happen within 1 or 2 hours after operation, meaning that if you are 5 years out of operation, I would not be concerned about infection anymore.

And the reason for that perforation was it occurred around the carina or the origin of the right upper bronchus because for the majority of the tracheomalacia, it occurs at the distal end of the trachea where you have to put the stent around the carinal portion. So if you make that stent a bit longer, the distal end of the stent would touch the origin of the bronchus. So any kind of migration would cause perforation of the prosthesis into the airway.

To avoid that, I have been using a little bit shorter graft, involving 3 to 4 rings instead of 5 to 6 . After starting this procedure, I never have seen this complication. All of these complications happened in the earlier series of ours. This is already a conquered problem I guess, I hope.
Dr Manning. The authors also conclude that the technique is effective for managing tracheobroncomalacia due to different underlying causes and that it may allow for airway growth. Both conclusions are well supported by their experience. Finally, they conclude that this technique may be a useful alternative or adjunct for dealing with severe airway malacia.

From your experience, Dr Ando, how do you currently stratify patients into the various treatment management strategies? Are there any findings on bronchoscopy, computed tomography scan, or your negative pressure testing that are helpful in guiding which patients will need external stenting rather than aortopexy alone?

Dr Ando. Thank you very much for that question. That is a very important issue.

First of all, I have to sort out that this stenting technique is only applicable to patients with malacia with vascular compression at either the trachea or main bronchus. So any kind of obstruction after the takeoff of the first branch would not be the objective treatment.

As for other procedures, I actually did aortopexy in some cases at the beginning of our series. In my hand, it works very well for the moment, but some percentage of patients came back for reoperation probably because, again, there are topographic changes in the relationship between the vessel and the airway that can cause reobstruction, or maybe the stitch can be loosened when the patient starts moving around.

So it was something that did not fulfill my expectations, I just switched to the external stenting at some point and found out that this is going to work for most of the cases. It is very reproducible, so I just stick to it. I have been doing this procedure exclusively for most of the cases, so I do not have any idea about another operation. Thank you very much.

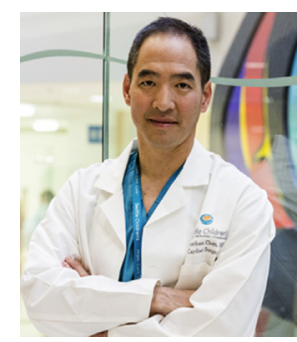

Dr Jonathan M. Chen (Seattle, Wash). It was a beautiful presentation. I actually just have a couple of quick, technical questions.

How do you choose the size of the graft with your experience now? Do you go for the biggest graft you can put in, or is there an algorithm based on weight or body surface area?

The second question is, your images looked as though you reinforced the base of the graft with polytetrafluoroethylene, and I was interested in how you evolved to that.

And the final question is, do you use bronchoscopy to decide where to put the sutures, and do you put them radially across the area of narrowing, or do you put them longitudinally across the entire trachea?

Dr Ando. Thank you very much for your questions. It is very important. The first was about the-what was the first question? 
Dr Chen. The first is how you choose the size.

Dr Ando. Yes, choosing the size of the graft. We do not have objective data, but from our experience, what we are dealing with is the age range of about let's say infant to age 3,4 , or 5 years. In those cases, you can instantly tell that it is impossible to put a 20 graft. So from our experience with small children, 16 is the biggest. Nothing more than that can be inserted.

Actually, I measured the diameter of the trachea or stent at the beginning of the operation. And in the big kids, probably the trachea can be as large as a centimeter, so in that case, 16 will be great.

But let's say the patient is $2.5 \mathrm{~kg}$, small infant, the bronchus could be as small as $5 \mathrm{~mm}$. So in that case, 16 would be huge, and I am sure that it is going to compress some other structure around it. So in that case, I set the minimum smallest size as 12 and try to use it as much as possible.

You are talking about the modification when I put in the single stitch? Yes, that's right. What I was afraid of was erosion of the graft into the vessel.

Dr Chen. Your images along the base of the cut edge, you have polytetrafluoroethylene.

Dr Ando. That's right. That's right. I was just afraid about cutting into the vessels or something else.

Dr Chen. Did the sutures go radially or longitudinally?

Dr Ando. Longitudinally.

Dr Chen. And do you use bronchoscopy?

Dr Ando. They use bronchoscopy, repeated 3 or 4 times during the operation, to make sure that the operation is going well.

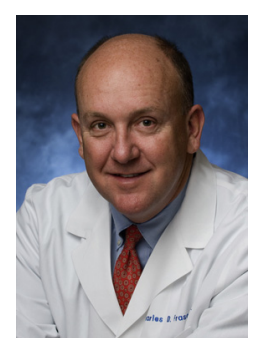

Dr Charles D. Fraser, Jr (Houston, Tex). Thank you for that presentation, beautifully done.

I just wanted to make sure I am understanding. This is a ringed polytetrafluoroethylene graft that is split?

Dr Ando. Correct.

Dr Fraser. And then you put a thin sheet of polytetrafluoroethylene behind on the membranous airway. Is that right?

Dr Ando. Usually, let's say we cut the graft and split it in half and sandwich the airway from one side and then the other. Then I sutured it to the cartilage portion and the membranous portion. It is overlapping like this, and it is not sutured together.

Dr Fraser. I got you.

Dr Ando. This is the standard technique.

Dr Fraser. And then while you are doing that, you are simultaneously working with your ear, nose, and throat colleagues or someone to bronchoscope and watch the dynamic airway change?

Dr Ando. Yes. I think that is the most important part. Our third author is a professional respiratory physician dedicated for the neonates. And these 98 patients were under either myself or the second author, almost 50 cases and 50 cases.

But for all of these cases, this third author, meaning the respiratory physician, was involved for the assessment of the success of the procedure.

So I think it is very crucial to have a professional take a look at the bronchus to evaluate the effectiveness of the stent.

Dr Fraser. Very nice. Thank you. 